\title{
THE VALIDITY OF CURRENT CLINICAL TESTS OF CONTRAST SENSITIVITY AND THEIR ABILITY TO PREDICT READING SPEED IN LOW VISION
}

\author{
SUSAN J. LEAT and GEORGE C. WOO \\ Waterloo, Ontario, Canada
}

\begin{abstract}
SUMMARY
Purpose: Contrast sensitivity (CS) testing using chart tests of $\mathrm{CS}$ is becoming increasingly common in low vision assessment. Yet we know little about the validity of these charts, i.e. which region of the spatial frequency spectrum is being measured. In this study we aimed to determine the validity of currently available CS charts by comparison against oscilloscope-based CS. We also determined their relative ability to predict reading speed.

Methods: CS was measured with five commercially available charts and the contrast sensitivity function was determined with sinusoidal gratings presented on a Joyce screen using a two-alternative forced choice staircase technique in 36 observers with low vision and 3 with normal vision. Reading rate was also measured with the subject reading with his or her own optical low vision aid.

Results: The results show that the Pelli-Robson chart and the Cambridge gratings are good measures of medium to low spatial frequencies, as would be predicted from their design, while the Regan and UW charts correlated with medium to high frequencies. The Vistech chart was a good predictor of CS at each spatial frequency.

Conclusions: The best chart test of CS depends on which region of the CS curve is of interest. All the charts were good predictors of reading rate.
\end{abstract}

It is becoming increasingly recognised that assessment of contrast sensitivity (CS) is an important aspect of a functional low vision assessment. CS at low spatial frequencies, around 0.5 cycles per degree (cpd), ${ }^{1,2}$ or peak $\mathrm{CS}^{3}$ have been shown to predict reading rate with magnification in subjects with low vision. Whittaker and Lovie-Kitchin ${ }^{4}$ summarise many of the studies which indicate that prediction of reading rates in low-vision observers can be made

Correspondence to: Susan J. Leat, Centre for Sight Enhancement, School of Optometry, University of Waterloo, Ontario N2L 3G1, Canada. Tel: +1 (519)-885-1211 ext 2040. Fax:+1 (519)-725-0784. from their CS. They describe the results in terms of contrast reserve (the ratio of the contrast of the print to the contrast threshold of the reader) and give the contrast reserves necessary to obtain certain reading speeds, once the print is magnified appropriately. The contrast reserve becomes decreased when the $\mathrm{CS}$, or the contrast of the letters, or both, is reduced. Since normal observers have a large contrast reserve (usually around $28: 1^{4}$ ) the contrast of the print can be significantly reduced before reading rate is severely affected. ${ }^{5}$ Alternatively, many observers with low vision are operating with a limited reserve and are thus far more sensitive to reduction in the contrast of the print. ${ }^{3}$ Aspects of CS not only predict reading performance, but also other aspects of daily life and perceived functioning: mobility, ${ }^{6}$ perception of faces, ${ }^{7}$ perceived disability, ${ }^{8,9}$ difficulty with daily tasks ${ }^{10}$ and glare sensitivity. ${ }^{11}$

Clinically, CS is measured with chart tests of CS, which are often composed of letters rather than gratings. Although the repeatability of these charts has been shown to be good, ${ }^{11-13}$ little is known of their validity in terms of which region of the spatial frequency spectrum is being measured. Inspection of Owsley et al.'s ${ }^{14}$ data seems to indicate that the Regan low contrast charts measure CS for medium to high spatial frequencies as would be expected from their design, being measures of low contrast acuity rather than strictly tests of CS. The University of Waterloo (UW) chart is similar. The Pelli-Robson chart would be expected to measure CS at approximately $1.3 \mathrm{cpd}$, if used at $1 \mathrm{~m}$, according to its design assumptions. ${ }^{15}$ The design was based on the work of Legge et al. ${ }^{16}$ who used a low-frequency band-pass filter to show that, as progressively higher to medium spatial frequencies were eliminated, reading speed started to be compromised once spatial frequencies of 2 cycles per character and lower were eliminated. Pelli and Robson assumed that detection of letters at contrast threshold is determined by CS at 2 cycles per character and the chart was composed of letters 
which subtended $0.5^{\circ}$ at $3 \mathrm{~m}$. Recent results from Leat and Munger ${ }^{17}$ indicate that this assumption may not always be valid. They applied a band-pass filter of varying widths and centre spatial frequency to text and showed that there is no specific spatial frequency necessary for reading text. Subjects were able to read text efficiently when only high spatial frequency components remained, or only medium or only low frequencies. The threshold bandwidth was, in fact, narrower when centred at intermediate to high spatial frequencies than at lower frequencies. This result causes us to question the assumption that, when at threshold, a letter is detected by contrast sensitivity channels at 2 cycles per character, since words are equally well detected by intermediate and high spatial frequency information.

In this study we investigated the validity of various commonly used chart tests of CS, using CS for sinusoidal gratings measured by presentation on an oscilloscope screen as the 'gold standard' to determine which region of the CS function is being measured with each chart. The main objective was to validate the letter charts of contrast sensitivity, but some other commonly used clinical tests were included, i.e. the Vistech chart and the Cambridge gratings. ${ }^{18}$ The former are printed sine-wave gratings, and the latter are dot-matrix printed squarewave gratings that subtend $4 \mathrm{cpd}$ at $6 \mathrm{~m}$.

In addition, since letter charts are frequently used in low vision practice to predict potential reading rate, we considered a second measure of validity, i.e. correlation between the chart tests of CS and reading rate.

\section{METHODS}

A total of 39 subjects took part. Thirty-six had low vision due to a heterogeneity of ocular disorders and were recruited from the Low Vision Clinic at the School of Optometry. Three subjects had normal visual acuity. The study was approved by the University of Waterloo Office of Research. The subjects were chosen to give a wide range of visual acuity (from normal to very reduced) as a way of attempting to obtain a wide range of $\mathrm{CS}$, but otherwise were randomly selected. The age range was from 41 to 90 years and the range in visual acuity was from $6 / 4.5$ to $1 / 48$. All the low vision subjects had previously been assessed for low vision aids in the Low Vision Clinic and were therefore considered to have the optimum low vision aid for their requirements. Subjects who were sensitive to increased illumination were generally prescribed internally illuminated stand magnifiers with a halogen light source. All subjects had been using this low vision aid for at least 5 months prior to this study and therefore can be expected to have completed the initial adaptation period to low vision aid usage. The visual acuity was remeasured and the refractive error was checked. All were corrected for refractive error during the testing, including the appropriate near addition for viewing the charts at $1 \mathrm{~m}$. The eye with better visual acuity or the eye normally used for reading was used while the fellow eye was occluded. The ocular diagnosis was known from the subject's file and this was confirmed by direct ophthalmoscopy. Table I shows the characteristics of the subjects.

Monocular contrast sensitivity was measured with the Pelli-Robson chart, Regan Low Contrast chart (3\% contrast), UW (University of Waterloo) Low Contrast chart $(10 \%$ contrast), Cambridge gratings and the Vistech chart. Test distance was $1 \mathrm{~m}$. The charts were used according to usual practice ${ }^{12}$ or the manufacturer's instructions (Cambridge gratings ${ }^{18}$ ). The UW and Regan charts were scored in logMAR acuity.

Additionally, contrast thresholds were determined using vertical sinusoidal gratings generated on a white phosphor Joyce screen and subtending $13^{\circ}$ by $9^{\circ}$, using a spatially separated, two alternative forced choice, double staircase procedure. ${ }^{19}$ The double staircase was for two different spatial frequencies interleaved. The staircase terminated when 40 stimulus presentations had been made, which resulted in at least 5 reversals. The total spatial extent of each grating was $6.5^{\circ}$ horizontally by $9^{\circ}$ vertically. Contrast sensitivity by this method was measured at $0.5,1,2,4$, and $8 \mathrm{cpd}$. For some subjects it was not possible to obtain a measure at the higher

Table I. Characteristics of subjects

\begin{tabular}{|c|c|c|c|c|}
\hline Ocular pathology & No. of subjects & $\begin{array}{l}\text { Mean age } \\
\text { (years) }\end{array}$ & $\begin{array}{l}\text { Mean VA } \\
(\log \text { MAR) }\end{array}$ & $\begin{array}{c}\text { Range of VA } \\
\text { (logMAR) }\end{array}$ \\
\hline Dry (atrophic) ARM & 16 & 77.1 & 0.84 & 0.14 to 1.66 \\
\hline Disciform ARM & 7 & 77.1 & 0.903 & 0.30 to 1.64 \\
\hline Diabetic retinopathy & 3 & 67.3 & 0.82 & 0.64 to 1.02 \\
\hline Optic atrophy & 2 & 55.5 & 0.92 & 0.64 to 1.20 \\
\hline RP & 2 & 61.5 & 0.68 & 0.34 to 1.02 \\
\hline RP inversus & 1 & 49 & 0.341 & \\
\hline High myopia & 1 & 43 & 1.103 & \\
\hline Achromatopsia & 1 & 41 & 1.04 & \\
\hline Macular hole & 1 & 83 & 1.0 & \\
\hline Chorioretinal atrophy & 1 & 54 & 1.12 & \\
\hline Normally sighted (no ocular pathology) & 3 & 59.7 & -0.03 & -0.13 to 0.04 \\
\hline
\end{tabular}

ARM, age-related maculopathy; RP, retinitis pigmentosa; VA, visual acuity. 
spatial frequencies as their CS was too poor. In these cases, CS was measured up to the highest spatial frequency possible. Luminance of all the tests was matched to approximately $100 \mathrm{~cd} / \mathrm{m}^{2}$.

Reading rate in correct words (units) per minute was measured using the Pepper test ${ }^{20}$ in $1.5 \mathrm{M}$ print, the subject reading with his or her own optical low vision aid and using the same eye as for the CS measurements. For those subjects who used spectacle microscopes, illumination was provided by an Anglepoise desk lamp. The Pepper test is composed of unrelated letters and words in increasing complexity throughout the test and has been specially designed to test the reading performance of subjects with central scotomata. The subjects read aloud, being requested to read as fast as possible, but not to sacrifice accuracy for speed. The number of errors, omissions, etc., were marked according the protocol described by Baldasare et $a .^{20}$ and used by Leat and Woodhouse. ${ }^{1}$ Correct units per minute were calculated. Two

A

Pelli-Robson

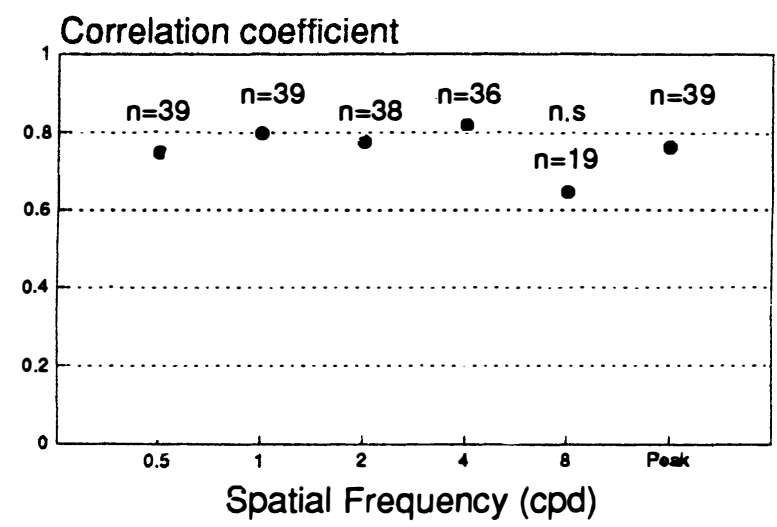

C

Regan Low Contrast

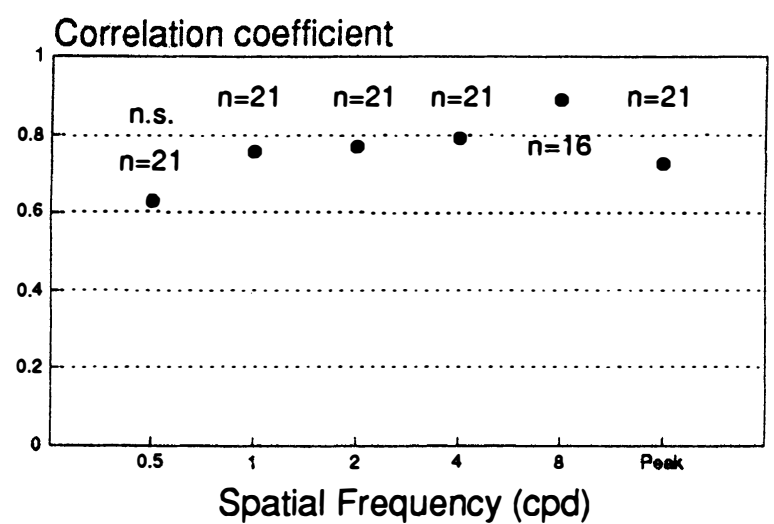

subjects were unable to read anything on the Pepper test (zero reading rate). Of these, one was unable to perform on the UW chart and they both obtained an identical low score on the Cambridge gratings.

All the data were converted to a logarithmic form (except for those already in log form, i.e. PelliRobson, UW and Regan charts).

\section{RESULTS}

For each chart test of CS, correlation coefficients were calculated for its score against CS at each spatial frequency as measured on the Joyce screen and also for CS at the peak of the CS curve, wherever that happened to be for each observer. Since a number of correlations were being performed, the significance level was adjusted to $0.05 / n$. They were found to be high and significant correlations between most measures of CS.

The results for the Pelli-Robson chart are shown in Fig. 1A in which the correlation coefficient is plotted

B

Cambridge Gratings

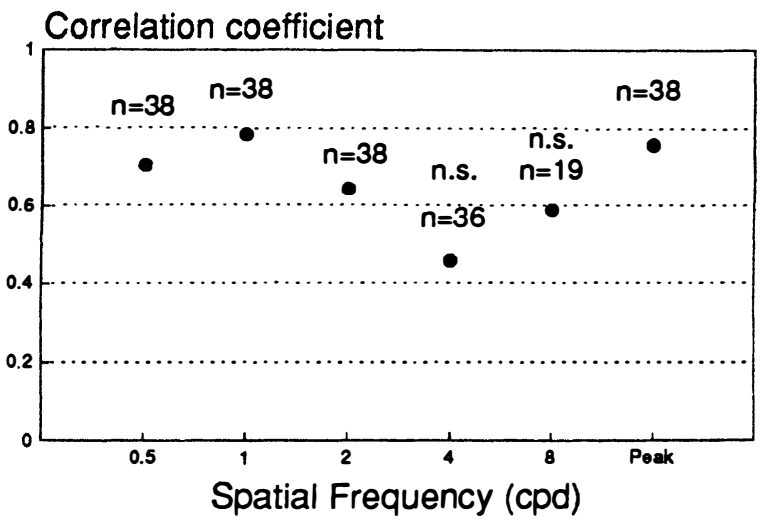

D

\section{UW Low Contrast}

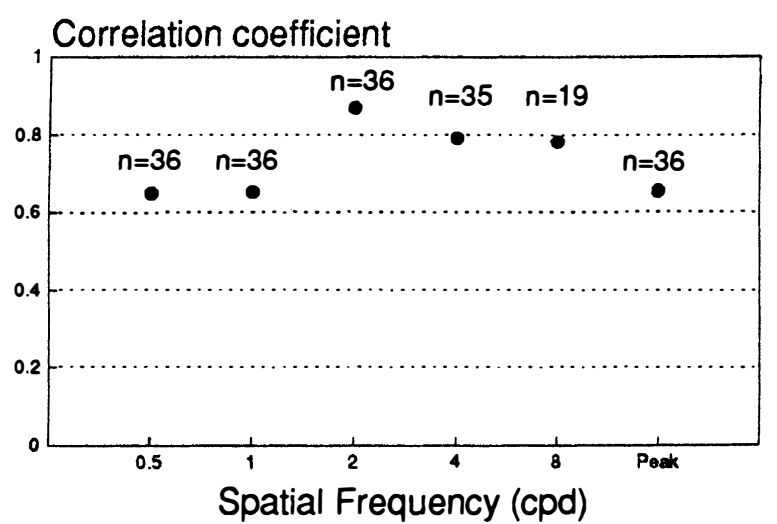

Fig. 1. Correlation coefficients are plotted for contrast sensitivity (CS) measured on each chart against CS as measured with sinusoidal wave gratings at each spatial frequency on the Joyce screen. The correlation with the peak of the CS curve is also shown. The numbers of subjects able to perform at each spatial frequency is indicated and the correlations that are insignificant at the $\mathrm{p}=0.0008$ level are shown as n.s. (a) Pelli-Robson chart, (b) Cambridge gratings, (c) Regan Low Contrast chart, (d) UW Low Contrast chart. 
Table II. Correlation coefficients of contrast sensitivity measured on the Vistech chart versus Joyce screen

\begin{tabular}{|c|c|c|c|c|c|}
\hline & Vistech 0.5 & Vistech 1.0 & Vistech 2.0 & Vistech 4.0 & Vistech 6.0 \\
\hline$\overline{C S} 0.5$ & $\begin{array}{l}0.701 * \\
n=38\end{array}$ & $\begin{array}{c}0.742 * \\
n=38\end{array}$ & $\begin{array}{l}0.770^{*} \\
n=37\end{array}$ & $\begin{array}{l}0.545(\mathrm{~ns}) \\
n=31\end{array}$ & $\begin{array}{l}0.434(\mathrm{~ns}) \\
n=20\end{array}$ \\
\hline CS 1.0 & $0.722 *$ & $0.797 *$ & $0.829 *$ & $0.633^{*}$ & 0.632 (ns) \\
\hline & $n=38$ & $n=38$ & $n=37$ & $n=31$ & $n=20$ \\
\hline CS 2.0 & $0.674^{*}$ & $0.750^{*}$ & $0.827 *$ & $0.760 *$ & $0.706^{*}$ \\
\hline CS 4.0 & $n=38$ & $n=38$ & $n=37$ & $n=31$ & $n=20$ \\
\hline CS 4.0 & $\begin{array}{l}0.567 * \\
n=36\end{array}$ & $\begin{array}{l}0.671 * \\
n=36\end{array}$ & $0.753^{*}$ & $\begin{array}{l}0.759 * \\
n=31\end{array}$ & $0.765^{*}$ \\
\hline CS 8.0 & $\begin{array}{l}n=36 \\
0.548(\mathrm{~ns})\end{array}$ & 0.673 (ns) & $\begin{array}{l}n=35 \\
0.846^{*}\end{array}$ & $\begin{array}{l}n=31 \\
0.771 *\end{array}$ & $\begin{array}{l}n=20 \\
0.856^{*}\end{array}$ \\
\hline & $n=19$ & $n=19$ & $n=19$ & $n=19$ & $n=15$ \\
\hline Peak CS & $\begin{array}{l}0.751^{*} \\
n=38\end{array}$ & $\begin{array}{l}0.822^{*} \\
n=38\end{array}$ & $\begin{array}{l}0.824^{*} \\
n=37\end{array}$ & $\begin{array}{l}0.620^{*} \\
n=31\end{array}$ & $\begin{array}{l}0.655(\mathrm{~ns}) \\
n=20\end{array}$ \\
\hline
\end{tabular}

*Significant at the $p=0.0008$ level. A high level of significance was taken because of the number of comparisons performed.

against CS at each spatial frequency as measured with the sine wave gratings. It can be seen that the highest correlation coefficients were obtained between 0.5 and $4 \mathrm{cpd}$, indicating that the PelliRobson chart is a good measure of CS at low and intermediate spatial frequencies. There was also a good correlation between the Pelli-Robson chart and peak CS. Similarly, the results of the Cambridge gratings are plotted in Fig. 1B. These also show maximum correlation with low spatial frequencies. Fig. 1C and D illustrate the results of the Regan and UW charts similarly plotted, and show that both these charts correlate optimally with medium to high spatial frequencies.

In Table II the results of the Vistech chart are shown. It can be seen that, for each spatial frequency, the maximum correlation coefficient was found at either the same spatial frequency or an adjacent spatial frequency as measured on the oscilloscope.

All the charts tests correlated with reading rate (Fig. 2), although the Regan chart did not reach significance due to the smaller number of subjects who were able to obtain a measure on this chart. The

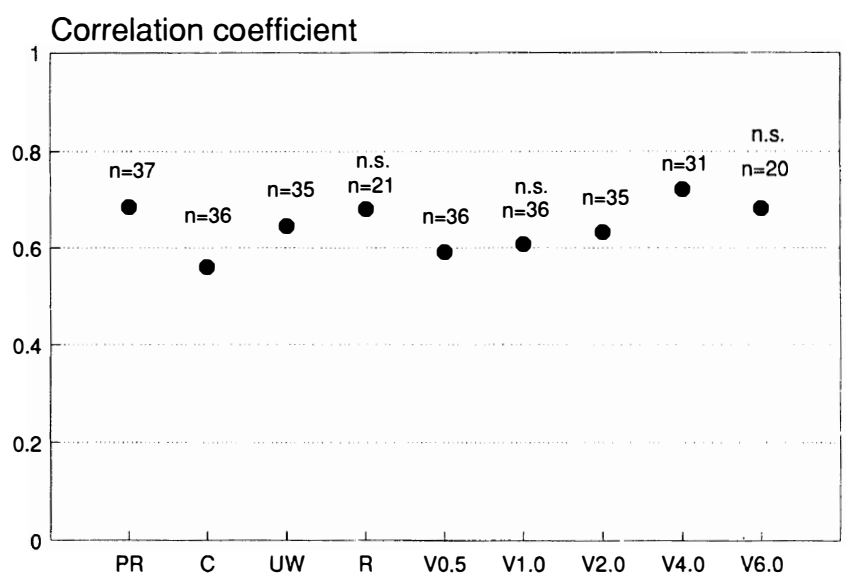

Fig. 2. Correlation of reading rate against contrast sensitivity as measured on each chart. PR, Pelli-Robson; $C$, Cambridge gratings; UW, UW Low Contrast chart; $R$, Regan Low Contrast chart; V0.5 to V6.0, Vistech chart at 0.5 to 6 cycles per degree. The numbers of subjects able to obtain a score are indicated. n.s., not significant at the 0.0008 level.
Pelli-Robson, UW and $4 \mathrm{cpd}$ Vistech charts were found to give the highest correlations with reading rate.

\section{DISCUSSION}

The finding that most of the charts correlate well with a range of spatial frequencies is not unexpected as (a) adjacent spatial frequencies are intercorrelated and (b) letters are a broad-band stimulus containing a range of spatial frequency components as are the Cambridge square wave gratings.

The results with the Pelli-Robson chart are consistent with the assumptions made in its design. If letters are detected at threshold by channels sensitive to 2 cycles per character, we would expect the highest correlation at $1-2 \mathrm{cpd}$, when the chart is used at $1 \mathrm{~m}$ rather than $3 \mathrm{~m}$, which is what this study confirms.

The Cambridge gratings were found to be a good estimate of CS at low spatial frequencies - as expected since the gratings are $0.66 \mathrm{cpd}$ square waves when the test is used at $1 \mathrm{~m}$. According to Fourier theory, a square wave is detected by whichever is the first component (fundamental or harmonic) to reach its own threshold. ${ }^{21}$ The first harmonic is at 3 times the frequency of the fundamental with one-third of its amplitude, while the second harmonic is at 5 times the frequency of the fundamental with one-fifth the amplitude. In the case of a $0.66 \mathrm{cpd}$ square wave, the first harmonic would be $2 \mathrm{cpd}$ and the second would be $3.3 \mathrm{cpd}$. Seventy-seven per cent of subjects had their peak CS at $1 \mathrm{cpd}$, while only $20 \%$ had the peak at $2 \mathrm{cpd}$ and one subject's CS peaked at $0.5 \mathrm{cpd}$. No subject had $\mathrm{CS}$ at $2 \mathrm{cpd}$ that was 3 times that at $1 \mathrm{cpd}$. Thus it seems that the majority of subjects will have detected the Cambridge gratings by the fundamental and not by one of the higher harmonics, which is consistent with the finding that the best correlation was between the Cambridge gratings and the lower spatial frequencies on the Joyce screen.

Both the UW and the Regan charts are strictly measures of low contrast visual acuity, rather than CS. Contrast remains constant and the resolution 
A

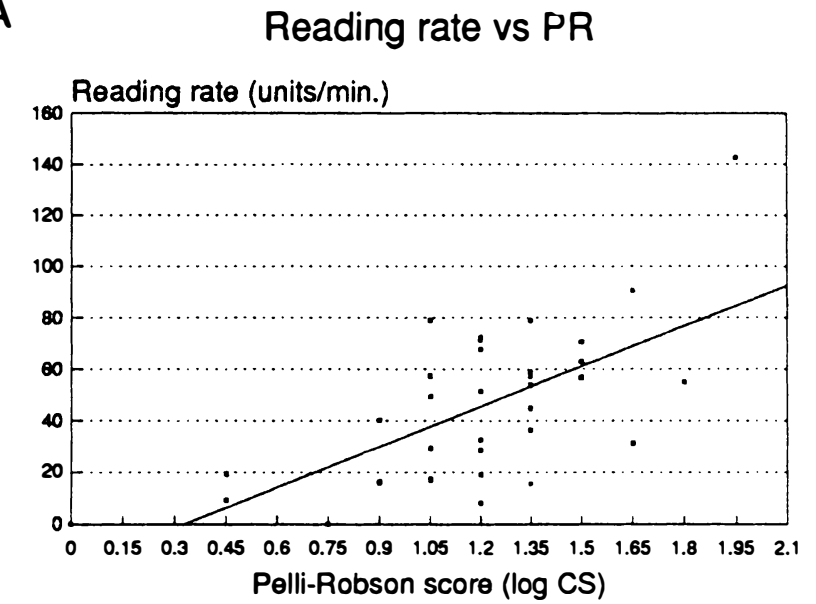

B

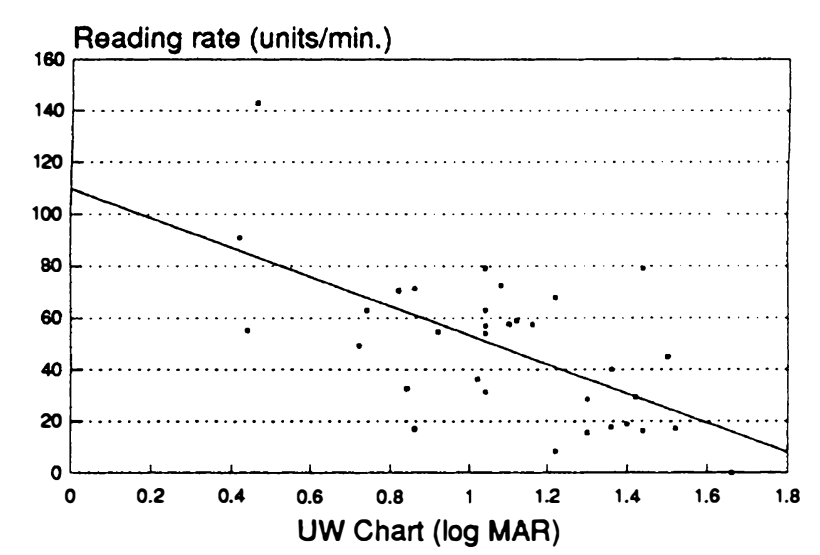

C

Reading rate vs CG

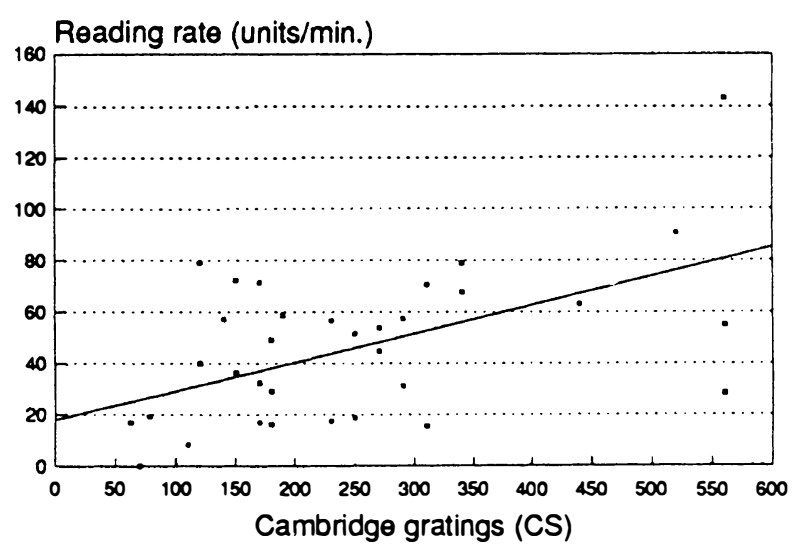

Fig. 3. Scatterplots of reading rate with the Pepper test against CS. Regression lines are shown. (a) Pelli-Robson chart, (b) UW chart, (c) Cambridge gratings.

limit at that contrast is determined. Therefore, we would expect that, if they correlate well with CS at all, it would be with the higher spatial frequencies, as has already been shown by Owsley et al. ${ }^{14}$ This was confirmed by the present results.

The Vistech chart was found to be a valid measure of CS at each spatial frequency.
The ideal chart for measuring CS clinically is one that is fast, repeatable and valid. CS is not one single entity. Indeed, CS losses can be mainly at high, high and intermediate, chiefly low, or all spatial frequencies. $^{22-27}$ The results shown here indicate that the optimum chart test of CS will depend on what region of the spatial frequency spectrum is of interest. Reading rates and mobility have been shown to correlate with low frequencies, and therefore to predict these functions, CS at low frequencies would be important. The present results indicate that the Pelli-Robson chart, the Cambridge gratings and the low frequencies of the Vistech would give this information. Of these, the Pelli-Robson chart has the best repeatability, ${ }^{11}$ the Cambridge gratings have questionable repeatability ${ }^{28}$ and the Vistech chart has particularly poor repeatability. ${ }^{11.12,29}$ The PelliRobson chart appears, therefore, the chart of choice for this task.

If CS at high frequencies is of interest, either the UW or Regan chart would be chosen. The Regan chart has good repeatability ${ }^{11}$ and the UW chart would be expected to have similar high repeatability, being of essentially similar construction. However, in the present study $18(50 \%)$ of the low vision subjects could not detect any letters on the 3\% contrast Regan chart and $3(8 \%)$ could not perform on the UW chart. This indicates that the higher contrast level $(10 \%)$ and the larger letters of the UW chart allow more low vision patients to obtain a score. Using the Regan $11 \%$ chart at a closer distance would have allowed more subjects to obtain a score.

The UW and Pelli-Robson charts were found to have the best predictive ability of reading rate ( $r=0.646, p=0.0003$ accounting for $42 \%$ of the variance and $0.685, p<0.00005$ accounting for $47 \%$ of the variance respectively). Scattergrams are shown of reading rate against $\mathrm{CS}$ for these charts in Fig. 3A and $\mathrm{B}$. In these figures only the results for low vision observers are included, since we will use them to predict reading rates of people with low vision. Although the Vistech chart at $4 \mathrm{cpd}$ was also found to correlate with reading rate, we will not consider it as a predictive test, because its poor repeatability makes it of questionable value. Since the Cambridge gratings may also be a useful test of CS in infants and other patients with communication difficulties who cannot respond to a letter test, we have also shown a scattergram of reading rate against CS measured with this (Fig. 3C). In each of these figures, the linear regression line is shown. Reading rates with the Pepper test, which is made up of unrelated letters and words, are approximately 2 times lower at lower reading rates than with text with contextual meaning, ${ }^{30}$ although there is less difference at higher reading rates. Whittaker and Lovie-Kitchin ${ }^{4}$ define high fluent, fluent and spot reading $(160,80$ and 40 
Table III. Contrast sensitivity that is predictive of spot, fluent or high fluent reading ${ }^{4}$

\begin{tabular}{lccc}
\hline \multirow{2}{*}{$\begin{array}{l}\text { Reading speed for continuous text in } \\
\text { words per minute (Pepper test) }\end{array}$} & Pelli-Robson chart & UW Low Contrast chart & Cambridge gratings \\
\cline { 2 - 4 } & 0.33 & 1.94 & NA \\
Spot reading $=40$ wpm $(22)$ & 0.75 & 1.55 & 35.5 \\
Fluent reading $=80$ wpm $(55)$ & 1.4 & 0.97 & 330 \\
High fluent $=160$ wpm $(117)$ & NA & NA & NA \\
\hline
\end{tabular}

The reading rates in words per minute (wpm) are given together with the equivalent reading rates with the Pepper test (units per minute). NA, not applicable.

words per minute), for continuous text. High fluent reading is probably necessary for reading a book or newspaper article, fluent reading would allow a person to read mail and letters, while spot reading is unlikely to allow more than identification of prices on price tags or addresses on an envelope and similar 'survival reading tasks'. We have taken the equivalent reading rates with the Pepper test and found the point of intersection on each regression line for each chart. Thus contrast sensitivity values that are predictive of each reading rate are shown in Table III with the addition of the CS that predicts zero reading rate.

From the scatterplots (Fig. 3) it can be seen that there is a large amount of scatter, particularly in the case of the Cambridge gratings. It may be more helpful to consider the results in terms of maximum achievable reading rate for a given CS value. By inspection of Fig. 3C it can be seen that there were no observers who had a CS of less than 120 who were able to read at faster than 20 words per minute. In order to achieve a reading rate of 55 units per minute (which is equivalent to fluent reading with continuous text), CS must be at least 120 . Similarly with the Pelli-Robson chart, to obtain fluent reading (55 units per minute) $\log$ CS must be at least 1.05 , and on the UW chart low contrast acuity must be $1.44 \log$ MAR or better.

\section{CONCLUSIONS}

All the chart tests of contrast sensitivity were found to have good validity when compared with CS measured with sinusoidal gratings presented on an oscilloscope using standard, criterion-free psychophysical methods. The letter charts of CS were generally measuring the aspects of CS that would be expected from their design.

All the charts were found to be good predictors of reading rate with magnification devices. However, since the Vistech chart is known to have poor repeatability, we recommend either the Pelli-Robson or the UW chart for predicting reading rate in low vision. The Pelli-Robson chart has good repeatability and the results were well correlated with reading rate. The UW chart is very similar in concept to the Regan charts, which also have good repeatability, and the UW chart also correlated well with reading rate. The Cambridge gratings were a poorer pre- dictor or reading speed and have been shown to have poorer repeatability than letter tests of CS, but could be used to give an estimate in children or adults who cannot read letters.

This study was supported in part by the Canadian Optometric Education Trust Fund and by the Burton Fund. We would like to thank all the subjects who took part in this study and Dennis Meyer for technical assistance.

Key words: Contrast sensitivity, Low vision, Low contrast acuity, Reading.

\section{REFERENCES}

1. Leat SJ, Woodhouse JM. Reading performance with low vision aids: relationship with contrast sensitivity. Ophthalmic Physiol Opt 1993;13:9-16.

2. Brown B. Reading performance in low vision patients: relation to contrast and contrast sensitivity. Am J Optom Physiol Opt 1981;58:218-26.

3. Rubin GS, Legge GE. Psychophysics of reading. VI. The role of contrast in low vision. Vision Res 1989;26: 79-91.

4. Whittaker GS, Lovie-Kitchin J. Visual requirements for reading. Optom Vis Sci 1993;70:54-65.

5. Legge GE, Rubin GS, Luebker A. Psychophysics of reading. $\mathrm{V}$. The role of contrast in normal vision. Vision Res 1987;27:1165-77.

6. Marron JA, Bailey IL. Visual factors and orientationmobility performance. Am J Optom Physiol Opt 1982; 59:413-26.

7. Owsley C, Sloane M. Contrast sensitivity, visual acuity and perception of 'real world' targets. Br J Ophthalmol 1987;71:791-6.

8. Lennerstrand G, Ahlstrom C. Contrast sensitivity in macular degeneration and the relation to subjective impairment. Acta Ophthalmol (Copenh) 1989;67: 225-33.

9. Elliott DB, Hurst MA, Weatherhill J. Comparing clinical tests of visual function with the patient's perceived disability. Eye 1990;4:712-7.

10. Rubin GS, Roche KB, Prasad-Rao, P, Fried LP. Visual impairment and disability in the elderly. Optom Vis Sci 1994;71:750-60.

11. Elliott DB, Bullimore MA. Assessing the reliability, discriminibility, and validity of disability glare tests. Invest Ophthalmol Vis Sci 1993;34:108-19.

12. Rubin GS. Reliability and sensitivity of clinical contrast sensitivity tests. Clin Vis Sci 1988;2:169-77.

13. Elliott DB, Sanderson K, Conkey A. The reliability of the Pelli-Robson contrast sensitivity chart. Ophthalmic Physiol Opt 1990;10:21-4.

14. Owsley C, Sloane ME, Skalka HW, Jackson CA. A comparison of the Regan low-contrast letter charts and contrast sensitivity testing in older patients. Clin Vis Sci $1990 ; 5: 325-34$. 
15. Pelli DG, Robson JG, Wilkins AJ. The design of a new letter chart for measuring contrast sensitivity. Clin Vis Sci 1988;2:187-99.

16. Legge G, Pelli DG, Rubin GS, Schleske MM. Psychophysics of reading. I. Normal vision. Vision Res 1985;25:239-52.

17. Leat SJ, Munger R. A new application of band-pass fast Fourier transforms to the study of reading performance. Technical Digest Series (Opt Soc Am) 1994;2:250-3.

18. Wilkins AJ, Della Sala S, Somazzi L, Nimmo-Smith I. Age-related norms for the Cambridge low contrast gratings, including details concerning their design and use. Clin Vis Sci 1988;2:202-12.

19. Woo GC, Campbell FW, Ing B. Effect of Fresnel prism dispersion on contrast sensitivity function. Ophthalmic Physiol Opt 1986;6:415-8.

20. Baldasare J, Watson G, Whittaker SG, Miller-Shaffer $\mathrm{H}$. The development and evaluation of a reading test for low vision individuals with macular loss. J Vis Impair Blind 1986;80:785-9.

21. Campbell FW, Robson JG. Application of Fourier theory to the visibility of gratings. J Physiol (Lond) 1968;197:551-66.
22. Hess R, Woo GC. Vision through cataracts. Invest Ophthalmol Vis Sci 1978;17:428-35.

23. Sjostrand J. Contrast sensitivity in macular disease using a small-field and a large-field TV system. Acta Ophthalmol (Copenh) 1978;57:832-46.

24. Loshin DS, White J. Contrast sensitivity: the visual rehabilitation of patients with macular degeneration. Arch Ophthalmol 1984;102:1303-6.

25. Ross JE, Bron AJ, Clarke DD. Contrast sensitivity and visual disability in chronic simple glaucoma. $\mathrm{Br} \mathrm{J}$ Ophthalmol 1984;68:821-7.

26. Regan D. Low-contrast letter charts and sinewave grating tests in ophthalmological and neurological disorders. Clin Vis Sci 1988;3:235-50.

27. Leguire LE. Do letter charts measure contrast sensitivity? Clin Vis Sci 1991;5:391-400.

28. Jones HS, Moseley MJ, Thompson JR. Reliability of the Cambridge Low Contrast Gratings. Ophthalmic Physiol Opt 1994;14:287-9.

29. Reeves BC, Wood JM, Hill AR. Vistech VCTS 6500 charts: within- and between-session reliability. Optom Vis Sci 1991;68:728-37.

30. Watson G, Baldasare J, Whittaker S. The validity and clinical uses of the Pepper Visual Skills for Reading test. J Vis Impair Blind 1990;84:119-23. 